\title{
ATRIBUTOS MICROBIOLÓGICOS, QUÍMICOS E FÍSICOS DE SOLO SOB DIFERENTES SISTEMAS DE MANEJO E CONDIÇÕES DE CERRADO ${ }^{1}$
}

\author{
Elaine Reis Pinheiro Lourente ${ }^{2}$, Fábio Martins Mercante 3 , Alessandra Mayumi Tokura Alovisi ${ }^{4}$, \\ Cezesmundo Ferreira Gomes ${ }^{4}$, Adriano Soares Gasparinii ${ }^{4}$, Cássio Miranda Nunes ${ }^{4}$
}

\section{ABSTRACT \\ MICROBIOLOGICAL, CHEMICAL AND PHYSICAL ATTRIBUTES OF SOIL UNDER DIFFERENT CROP SYSTEMS AND CERRADO CONDITIONS}

The aim of this research was to evaluate the effect of soil use and management on its chemical, physical and microbiological attributes. The research was carried out from the winter (2007) to the summer (2008), in Dourados, Mato Grosso do Sul State, Brazil, in a Distroferric Red Latossol. Data were analyzed as a complete randomized experimental design, with 5 collected samples that established 5 replications per management system. The management systems were: Area 1: area alloted to reforestation; Area 2: conventional soil tillage; Area 3: no-tillage; Area 4: degraded forage; and Area 5: natural system (native forest). The evaluation of a management system in its first year of implementation was not sufficient to evaluate the impacts on soil physical and microbiological attributes. The substitution of native vegetation by cultivation systems can cause important alterations on soil chemical attributes from the first year of implementation. Microbiological attributes were efficient indicators of alterations in physical attributes according to the soil use and management.

KEY-WORDS: Soil quality; microbial biomass carbon; management practices.

\section{INTRODUÇÃO}

O manejo agrícola é sustentável somente quando a qualidade dos recursos solo, ar e água é mantida ou melhorada, e, no caso do solo, a qualidade depende da manutenção e melhoria de seus atributos físicos, químicos e biológicos, bem como de sua contínua capacidade de produzir alimentos e fibras (Doran \& Parkin 1994).

Os solos sob Cerrado, no Brasil, em geral, apresentam condições físicas favoráveis à agricul-

\section{RESUMO}

O objetivo deste trabalho foi avaliar o efeito do uso e manejo de solo sobre seus atributos químicos, físicos e microbiológicos. A pesquisa foi realizada no inverno de 2007 e verão de 2008, no Município de Dourados (MS), em Latossolo Vermelho distroférrico típico. Os dados foram analisados de acordo com delineamento experimental inteiramente casualizado, sendo coletadas 5 amostras compostas, que constituíram 5 repetições por sistema de manejo. Os sistemas de manejo estudados foram: Área 1: área destinada a reflorestamento; Área 2: sistema convencional de preparo do solo; Área 3: sistema de semeadura direta; Área 4: pastagem degradada; e Área 5: sistema natural (mata nativa). A avaliação de um sistema de manejo, em seu primeiro ano de implantação, não foi suficiente para mensurar os impactos sobre atributos físicos e microbiológicos do solo. A substituição da vegetação nativa por sistemas de cultivo pode causar importantes alterações nos atributos químicos do solo, já no primeiro ano de implantação. Os atributos microbiológicos foram eficientes indicadores de alterações nos atributos físicos, em função do uso e manejo do solo.

PALAVRAS-CHAVE: Qualidade do solo; carbono da biomassa microbiana; práticas de manejo.

tura e vêm sendo, gradativamente, explorados com culturas anuais, pastagens e, mais recentemente, reflorestamentos. A mudança da vegetação natural para sistema de exploração agropecuária provoca alterações profundas nos atributos do solo. Quando uma área de vegetação nativa de Cerrado, por exemplo, é convertida em pastagem, ou área de cultivo de grãos, os atributos químicos e microbiológicos do solo são alterados (Costa et al. 2006, Carneiro et al. 2009).

1. Trabalho recebido em dez./2009 e aceito para publicação em jan./2011 ( ${ }^{\circ}$ registro: PAT 8459/ DOI 10.5216/pat.v41i1.8459).

2. Universidade Federal da Grande Dourados, Faculdade de Ciências Agrárias, Departamento de Engenharia Agrícola,

Dourados, MS, Brasil. E-mail: elainelourente@ufgd.edu.br.

3. Embrapa Agropecuária Oeste, Dourados, MS, Brasil.E-mail: mercante@cpao.embrapa.br.

4. Faculdades Anhanguera de Dourados, Departamento de Agronomia, Dourados, MS, Brasil.E-mails: agronomia.dourados@, unianhanguera.edu.br, cezes@hotmail.com, adriano_gasparini@yahoo.com.br, cassiomirandanunes@hotmail.com. 
Ocorrem, ainda, efeitos diferenciados sobre os atributos físicos, em função do tipo de preparo de solo adotado em cada sistema de manejo, e estes são dependentes da intensidade de revolvimento, trânsito de máquinas, tipo de equipamento utilizado, manejo de resíduos vegetais e das condições de umidade do solo, no momento do preparo (Vieira \& Muzilli 1984, Costa et al. 2006). O manejo incorreto de máquinas e equipamentos agrícolas leva à formação de camadas subsuperficiais compactadas. De acordo com Carneiro et al. (2009), este manejo irá acarretar interferência na estrutura do solo, promovendo redução do volume total de poros. A degradação da estrutura do solo pode reduzir a produtividade das culturas.

Uma importante alternativa para a manutenção da qualidade do solo é a manutenção de resíduos em sua superfície, que contribuem para a melhoria na sua estruturação (Calegari 2000). As respostas podem estar relacionadas ao aumento da estabilidade dos agregados (ação cimentante da matéria orgânica, efeito dos polissacarídeos e hifas de fungos), aumento da capacidade de retenção de água, aumento da porosidade, melhor aeração, menores perdas de água por evaporação e diminuição da densidade, pelo efeito da matéria orgânica.

Com relação à importância da biomassa microbiana nos atributos físicos do solo, Bayer \& Mielniczuk (1999) citam que os micro-organismos heterotróficos do solo liberam polissacarídeos de origem microbiana, durante a decomposição da matéria orgânica, que são mais persistentes no solo, quando comparados aos polissacarídeos de origem vegetal, desempenhando relevante papel na estabilidade de agregados. Segundo estes autores, além dos polissacarídeos microbianos, as hifas dos fungos estão ligadas à formação e estabilidade dos agregados.

Neste contexto, objetivou-se, com este trabalho, avaliar o efeito do uso e manejo sobre atributos químicos, físicos e microbiológicos do solo.

\section{MATERIAL E MÉTODOS}

A pesquisa foi realizada no inverno de 2007 e verão de 2008, na Fazenda Escola pertencente às Faculdades Anhanguera, no município de Dourados, MS (22 $14^{\circ} \mathrm{S}, 54^{\circ} 49^{\prime} \mathrm{W}$ e altitude de $\left.452 \mathrm{~m}\right)$. O solo da área experimental é classificado como Latossolo Vermelho distroférrico típico, originalmente sob vegetação de mata. O clima da região é caracterizado como Cwa (Köppen), subtropical, com chuvas de verão e verões quentes (Ayoade 1986) (Figura 1).
Foi desenvolvido um estudo de caso, sob diferentes sistemas de uso do solo. A área em que foram implantados os diferentes sistemas de manejo foi cultivada, anteriormente, sob sistema plantio direto, sendo a soja (Glycine $\max$ (L.) Merrill) a cultura de verão e a aveia preta (Avena strigosa Scheib) a cultura de inverno. Entretanto, nos últimos dois anos, a cultura de inverno foi constituída pelo nabo forrageiro (Raphanus sativus L.) e milho "safrinha" (Zea mays L.). As áreas estudadas foram: Área 1: destinada a reflorestamento $(\mathrm{R})$ com espécies nativas do Cerrado, sendo preparada com uma aração e duas gradagens e deixada em pousio por seis meses, tendo como vegetação predominante, no momento da coleta, o capim carrapicho (Cenchrus echinatus L.); Área 2: culturas anuais em monocultivo, sendo o primeiro ano sob sistema convencional de preparo do solo (SC) e a área preparada com uma aração e duas gradagens, com a coleta de solo realizada após o plantio de milho de inverno (safrinha); Área 3: culturas anuais em Semeadura Direta (SD), em área sem revolvimento há dois anos e cultivada, neste período, com a sucessão soja/milho; Área 4: área de pastagem degradada (P), sem correção do solo há 15 anos, cultivada com braquiária (Brachiaria decumbens Stapf), em avançado estádio de degradação, refletido em exposição do solo; e Área 5: sistema natural, com vegetação nativa (VN) adjacente à área experimental, utilizada como padrão comparativo das análises microbiológicas, químicas e físicas. Em cada sistema de manejo, foram retiradas cinco amostras compostas, à profundidade de $0-0,1 \mathrm{~m}$, cada uma constituindo uma repetição.

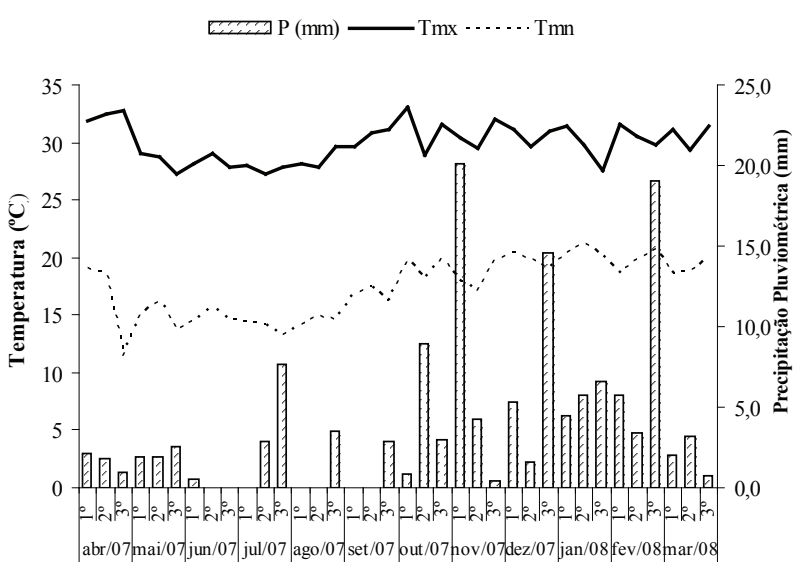

Figura 1. Precipitação pluviométrica acumulada e temperatura média, por descêndio, registradas na Estação Meteorológica da Fazenda Experimental da UFGD, entre abril de 2007 e março de 2008 (Dourados, MS). 
As análises biológicas foram realizadas no laboratório de solos da Embrapa Agropecuária Oeste, localizado em Dourados (MS). A análise do carbono da biomassa microbiana do solo (C-BMS) foi realizada pelo método da fumigação-extração, adotando-se fator de correção para eficiência de extração $\left(\mathrm{k}_{\mathrm{ec}}\right)$ igual a 0,33 (Vance et al. 1987); o C orgânico foi determinado pelo método de Mebius, modificado por Yeomans \& Bremner (1989); a respiração basal $\left(\mathrm{C}-\mathrm{CO}_{2}\right)$ foi obtida pelo método da respirometria (evolução de $\mathrm{CO}_{2}$ ); o quociente microbiano ( $q \mathrm{MIC}$ ), expresso em percentagem, foi calculado pela fórmula (C-BMS/Corg) x 100; e o quociente metabólico $\left(q \mathrm{CO}_{2}\right)$ foi obtido pela divisão dos valores da respiração basal pelo do carbono microbiano $\left(\mu \mathrm{CO}_{2} /\right.$ $\mu \mathrm{g} \mathrm{C}$-BMS $\left.\mathrm{h}^{-1}\right)$. As análises químicas do solo foram determinadas de acordo com metodologia descrita por Claessen (1997).

Os atributos físicos estudados foram analisados no laboratório de solos das Faculdades Anhanguera, determinando-se a granulometria, diâmetro médio ponderado (DMP) e volume total de poros (VTP). A análise granulométrica foi efetuada pelo método da pipeta, após dispersão da amostra com $\mathrm{NaOH} 1,0 \mathrm{~mol} \mathrm{~L}^{-1}$ e agitação rápida $(6.000 \mathrm{rpm})$, por 15 minutos (Claessen 1997).

A estabilidade dos agregados foi determinada utilizando-se o peneiramento em água, após pré-umedecimento lento por capilaridade, sobre papel-filtro umedecido. Para a separação das classes de tamanho dos agregados, foram utilizadas peneiras com malhas de $2 \mathrm{~mm} ; 1 \mathrm{~mm}$; $0,5 \mathrm{~mm}$; $0,25 \mathrm{~mm}$; e $0,105 \mathrm{~mm}$, submetidas a agitação em aparelho de Yooder, durante 15 minutos. Após o tempo determinado, o material retido em cada peneira, separadamente, foi colocado em recipientes metálicos, previamente pesados e identificadas, mantidos em estufa $\left(105^{\circ} \mathrm{C}\right)$, por 24 horas. Para se obter o diâmetro médio ponderado (DMP), foi utilizada a seguinte equação:

$$
\mathrm{DMP}=\sum_{i=1}^{a}(x i w i)
$$

em que $\mathrm{DMP}=$ diâmetro médio ponderado $(\mathrm{mm}) ; \mathrm{xi}=$ diâmetro médio das classes $(\mathrm{mm})$ e wi = proporção de cada classe, em relação ao total (Claessen 1997).

A determinação da densidade do solo foi realizada pelo método do anel volumétrico (Blake \& Hartge 1986), sendo coletadas amostras indeformadas com amostrador de Uhland, em cilindros com volume médio de $313,9 \mathrm{~cm}^{3}$. A porosidade total
$(\mathrm{VTP})$ foi calculada pela fórmula $\mathrm{Pt}=(\mathrm{Dp}-\mathrm{Ds} / \mathrm{Dp}) \mathrm{x}$ $100(\%)$.

Os dados foram analisados de acordo com o modelo de delineamento experimental inteiramente casualizado, com os tratamentos dispostos em esquema fatorial $5 \times 2$, sendo cinco sistemas de manejo e dois períodos (inverno e verão). Os dados foram submetidos a análise de variância e, quando significativos, os efeitos dos manejos e usos do solo foram analisados pelo teste de Duncan $(\mathrm{p} \leq 0,05)$. As variáveis independentes foram comparadas entre si, por meio da correlação de Pearson, sendo os efeitos testados pelo teste t, a 5\% e 1\%. Foi utilizado o aplicativo computacional SAEG 9.0 (Ribeiro Júnior 2001).

\section{RESULTADOS E DISCUSSÃO}

Os teores de carbono da biomassa microbiana do solo (C-BMS), nos sistemas de uso do solo, variaram entre 587,5 $\mu \mathrm{g} \mathrm{C} \mathrm{g}^{-1}$ e 253,3 $\mu \mathrm{g} \mathrm{C} \mathrm{g}^{-1}$ de solo seco, no inverno, e de $882,7 \mu \mathrm{g} \mathrm{C} \mathrm{g}^{-1}$ a $448,0 \mu \mathrm{g} \mathrm{C} \mathrm{g}^{-1}$ de solo seco, no verão, sendo, significativamente, maiores no verão (71\%, em média) (Tabela 1$)$. O período de verão caracteriza-se por apresentar maior temperatura e precipitação pluviométrica, enquanto, no inverno, as chuvas são escassas e as temperaturas médias são inferiores a $15^{\circ} \mathrm{C}$ (Figura 1).

De acordo com Piao et al. (2000), durante a estação seca, parte da biomassa microbiana morre e, com a retomada das chuvas e incremento da umidade do solo, a biomassa sobrevivente utiliza matéria orgânica acumulada no período, incluindo as células mortas, havendo, desta forma, maior atividade microbiana, durante o período chuvoso. O efeito da temperatura também deve ser considerado, uma vez que a elevação da temperatura do ar e da precipitação pluviométrica, no verão, acarretam condições favoráveis ao aumento da biomassa microbiana do solo (Espíndola et al. 2001).

A substituição da vegetação nativa por sistemas de cultivo causou importante redução média no C-BMS, da ordem de 56,89\%; 50,33\%; 46,93\%; e $40,14 \%$, no $\mathrm{SC}, \mathrm{PD}, \mathrm{R}$ e $\mathrm{P}$, respectivamente, no inverno, e de $49,25 \% ; 42,72 \%$; e $29,44 \%$, respectivamente, no verão. Apesar destes resultados, não houve diferença estatística nos valores desta variável, entre os diferentes sistemas, com interferência antrópica (Tabela 1). A diversidade de espécies da vegetação nativa (quantidade e qualidade) implica na deposição contínua de substratos orgânicos com composição 
Tabela 1. Variação de atributos microbiológicos de um Latossolo Vermelho distroférrico, em função do uso e manejo, no período de inverno e verão (Dourados, MS, 2007/2008).

\begin{tabular}{|c|c|c|c|c|c|c|c|c|}
\hline \multirow[t]{2}{*}{ Manejo e uso do solo } & \multicolumn{2}{|c|}{$\begin{array}{l}\text { C-BMS } \\
\left(\mu \mathrm{g} \mathrm{C} \mathrm{g}^{-1}\right)\end{array}$} & \multicolumn{2}{|c|}{$\begin{array}{c}\mathrm{C}-\mathrm{CO}_{2} \\
\left(\mu \mathrm{g} \mathrm{g}^{-1} \mathrm{dia}^{-1} \mathrm{de}^{2}-\mathrm{CO}_{2}\right)\end{array}$} & \multicolumn{2}{|c|}{$\begin{array}{c}q \mathrm{CO}_{2} \\
\left(\mu \mathrm{g} \mathrm{CO}_{2} / \mu \mathrm{gC}_{\text {mic }} \mathrm{h}^{-1}\right)\end{array}$} & \multicolumn{2}{|c|}{$\begin{array}{c}q \mathrm{MIC} \\
(\%)\end{array}$} \\
\hline & Inverno & Verão & Inverno & Verão & Inverno & Verão & Inverno & Verão \\
\hline Vegetação nativa (VN) & $587,5 \mathrm{Ba}^{*}$ & $882,7 \mathrm{Aa}$ & $29,0 \mathrm{Ba}$ & $33,9 \mathrm{Aa}$ & $21,4 \mathrm{Aa}$ & $16,7 \mathrm{Aa}$ & $1,62 \mathrm{Ba}$ & $2,8 \mathrm{Aa}$ \\
\hline Pastagem $(\mathrm{P})$ & $351,7 \mathrm{Bb}$ & $622,8 \mathrm{Ab}$ & $8,3 \mathrm{Bb}$ & $17,3 \mathrm{Ab}$ & $8,1 \mathrm{Ab}$ & $14,0 \mathrm{Aa}$ & $1,78 \mathrm{Ba}$ & $2,2 \mathrm{Aa}$ \\
\hline Semeadura direta (SD) & $291,8 \mathrm{Bb}$ & $505,6 \mathrm{Ab}$ & $6,2 \mathrm{Bb}$ & $14,9 \mathrm{Ab}$ & $9,0 \mathrm{Ab}$ & $14,1 \mathrm{Aa}$ & $1,61 \mathrm{Ba}$ & $2,2 \mathrm{Aa}$ \\
\hline Reflorestamento (R) & $311,8 \mathrm{Bb}$ & - & $5,9 \mathrm{Bb}$ & - & $11,4 \mathrm{Ab}$ & - & $1,72 \mathrm{Ba}$ & - \\
\hline Sistema Convencional (SC) & $253,3 \mathrm{Bb}$ & $448,0 \mathrm{Ab}$ & $4,7 \mathrm{Bb}$ & $14,8 \mathrm{Ab}$ & $9,7 \mathrm{Ab}$ & $12,6 \mathrm{Aa}$ & $1,54 \mathrm{Ba}$ & $2,7 \mathrm{Aa}$ \\
\hline Média & 359,2 & 614,8 & 11 & 21 & 11,9 & 14,4 & 1,7 & 2,5 \\
\hline $\mathrm{CV} \%$ & \multicolumn{2}{|c|}{29,0} & \multicolumn{2}{|c|}{20,0} & \multicolumn{2}{|c|}{34,0} & \multicolumn{2}{|c|}{32,0} \\
\hline
\end{tabular}

* Médias seguidas da mesma letra minúscula, na coluna, e maiúscula, na linha, não diferem entre si, pelo teste de Duncan ( $\mathrm{p} \leq 0,05)$. C-BMS $=$ carbono da biomassa microbiana ( $\mu \mathrm{g} \mathrm{C} \mathrm{g} \mathrm{g}^{-1}$ de solo); $q \mathrm{CO}_{2}=$ quociente metabólico $\left(\mu \mathrm{CO}_{2} / \mu \mathrm{g} \mathrm{C}-\mathrm{BMS} \mathrm{h}^{-1}\right) ; \mathrm{C}^{-\mathrm{CO}_{2}}=\mathrm{CO}_{2}$ respiração microbiana; $q \mathrm{MIC}=$ quociente microbiano $((\mathrm{C}-\mathrm{BMS} /$ Corg) $\mathrm{x} 100)$.

variada na serapilheira, que favorece maiores teores de biomassa microbiana (Correia \& Andrade 1999, D`Andréa et al. 2002, Matsuoka et al. 2003).

Comparando-se os sistemas plantio direto e convencional de preparo do solo, observou-se que não houve diferença significativa entre os sistemas, com relação aos valores do C-BMS. Geralmente, observa-se maior biomassa microbiana no plantio direto, quando comparado ao plantio convencional, em função do não revolvimento do solo, no primeiro sistema, o que provê constante suprimento de $\mathrm{C}$ orgânico à biomassa microbiana do solo, além de favorecer a maior agregação e estabilização dos agregados, habitat da microbiota (Balota et al. 2003, Costa et al. 2006). Já no sistema convencional de preparo do solo, há redução da atividade microbiana com o tempo de preparo, em função das perdas de $\mathrm{C}$, na forma de $\mathrm{CO}_{2}$ (Balota et al. 2003).

A semelhança entre o C-BMS, nestes dois sistemas, pode estar associada ao fato de a coleta de solo ter sido realizada no primeiro ano de plantio convencional, sendo que este foi, anteriormente, cultivado sob plantio direto, por dez anos. Resultados discordantes deste estudo foram observados por Benitende et al. (2008), os quais verificaram que o sistema com revolvimento do solo apresentou menor C-BMS, segundo estes autores, por consequência do impacto negativo do preparo do solo sobre as populações de micro-organismos do solo.

O solo sob vegetação nativa apresentou maior $\mathrm{C}-\mathrm{CO}_{2}$, quando comparado aos demais sistemas de manejo. Os valores observados foram de $29 \mu \mathrm{g} \mathrm{g}^{-1} \mathrm{dia}^{-1}$ e $33,9 \mu \mathrm{g} \mathrm{g}^{-1} \mathrm{dia}^{-1}$ de C-CO ${ }_{2}$ no solo, no inverno e verão, respectivamente (Tabela 1). A substituição da vegetação nativa pelos sistemas de cultivo promoveu redução na liberação de $\mathrm{CO}_{2}$ de $83,79 \% ; 79,6 \% ; 78,62 \%$; e 71,38\%, no SC, R, SD e $\mathrm{P}$, respectivamente, no inverno, e de $56,34 \% ; 56,05 \%$; e 48,97\%, no SC, SD e P, respectivamente, no verão. Este resultado, provavelmente, está relacionado aos maiores teores de carbono na biomassa, observados no solo sob vegetação nativa, quando comparados aos demais sistemas e à observação de uma correlação positiva e significativa entre C-CBMS e C-CO $(\mathrm{r}=$ 0,65*) (Matsuoka et al. 2003, Araújo et al. 2007).

A taxa de respiração basal $\left(\mathrm{C}-\mathrm{CO}_{2}\right)$, nos sistemas de uso de solo estudados, foi, em média, 53\% maior, no verão (Tabela 1). Resultados semelhantes foram obtidos por outros autores, que atribuem este fato à íntima relação entre $\mathrm{C}-\mathrm{CO}_{2}, \mathrm{C}-\mathrm{CBMS}$ e umidade do solo, que favorece a população e respiração microbiana (Alvarez et al. 1995, Espíndola et al. 2001). Os maiores índices pluviométricos ocorreram no verão e a umidade do solo é um fator relevante sobre a atividade microbiana, regulando-a de várias formas: como componente do protoplasma, alterando as trocas gasosas e atuando no transporte e dissolução dos nutrientes do solo (Alexander 1977).

Quanto ao quociente metabólico $\left(q \mathrm{CO}_{2}\right)$, somente foi observada diferença entre a vegetação nativa e os demais sistemas de uso do solo no inverno $\left(21,4 \mu \mathrm{g} \mathrm{CO}_{2} / \mu \mathrm{gC}_{\text {mic }} \mathrm{h}^{-1}\right)$, enquanto, no verão, não houve diferença significativa entre os sistemas de uso, com redução do $q \mathrm{CO}_{2}$ para vegetação nativa e incremento para os sistemas de cultivos (Tabela 1). A redução nos valores do $q \mathrm{CO}_{2}$, para a vegetação nativa, no verão, indica que a biomassa microbiana está sendo mais eficiente, ou seja, está havendo menor perda de $\mathrm{CO}_{2}$ por unidade de biomassa. À medida que determinada biomassa microbiana se torna mais 
eficiente, menos carbono é perdido como $\mathrm{CO}_{2}$ pela respiração e fração significativa de $\mathrm{C}$ é incorporada ao tecido microbiano (Gama-Rodrigues 1999).

$\mathrm{O}$ aumento de $\mathrm{C}-\mathrm{CO}_{2}$ e $q \mathrm{CO}_{2}$, observado no verão, indica que a biomassa microbiana estaria atuando na decomposição da matéria orgânica do solo, com imobilização de nutrientes em sua biomassa e liberação de parte destes nutrientes para a solução do solo. Menores valores no inverno sugerem que a biomassa microbiana está atuando como compartimento de reserva de nutrientes, nestes períodos, evitando-se perdas (Espíndola et al. 2001).

O quociente microbiano $(q \mathrm{MIC})$ representa a relação C-BMS/Corg e, neste contexto, solos que exibem valores maiores ou menores poderiam expressar ocorrência, respectivamente, de acúmulo ou perda de C, no solo (Balota et al. 1998). Nesta pesquisa, observou-se que o maior acúmulo de carbono da biomassa ocorreu no verão, sendo, significativamente, maior do que no inverno, nos diferentes sistemas de uso ou manejo do solo (Tabela 1). Resultados semelhantes foram observados por Lourente (2007), ao estudar atributos microbiológicos, em diferentes sistemas de manejo, em condições de Cerrado e sob Latossolo Vermelho distroférrico muito argiloso. Valores superiores a $1 \%$ desta relação indicam acréscimos de carbono ao solo, ao longo do tempo (Marchiori Júnior \& Melo 2000).

Independentemente do período de avaliação, não houve efeito dos sistemas de manejo sobre o $q \mathrm{MIC}$ (Tabela 1). A relação entre carbono microbiano e carbono orgânico ( $q \mathrm{MIC})$ é um indicador de disponibilidade da matéria orgânica para os micro-organismos, e valores maiores deste quociente indicam incremento da sua dinâmica no solo (Mercante 2001, Sampaio et al. 2008). De acordo com Alvarenga et al. (1999), a ausência de efeito dos sistemas de uso e manejo do solo sobre os valores desta variável in- dica ausência de alteração no equilíbrio ecológico do solo, em relação ao ecossistema natural de Cerrado. Estes resultados podem estar associados ao fato de a implantação dos diferentes sistemas de manejo ter ocorrido há apenas um ano.

Quanto aos atributos químicos do solo, a menor fertilidade foi observada na pastagem degradada $(\mathrm{p} \leq 0,05)$, representada pelos menores valores de $\mathrm{pH}$, $\mathrm{Ca}, \mathrm{CTC}, \mathrm{V}(\%)$ e elevada saturação em alumínio $(\mathrm{m} \%=19)$. Considerando-se os sistemas de preparo do solo, observou-se que o solo sob o sistema convencional de manejo, em relação ao plantio direto, apresentou médias estatisticamente menores, para as variáveis $\mathrm{pH}, \mathrm{MO}, \mathrm{Ca}, \mathrm{K}, \mathrm{SB}, \mathrm{CTC}$ e V\%, sendo que, sob plantio direto, o solo apresentou fertilidade adequada, à exceção do teor de fósforo, que foi muito abaixo daquele observado na vegetação nativa, reflorestamento e plantio convencional (Tabela 2). O maior teor de nutrientes, no sistema plantio direto, pode estar associado ao não revolvimento do solo, à manutenção de cobertura de resíduos de culturas sobre o solo e à reciclagem dos nutrientes pelas plantas (Falleiro et al. 2003).

$\mathrm{O}$ teor de fósforo foi significativamente menor na pastagem e $\mathrm{PD}$, quando comparado aos demais sistemas de uso e manejo do solo. $\mathrm{O}$ teor de $\mathrm{P}$ observado na pastagem pode estar associado à ausência de reposição de nutrientes e correção do solo, há, pelo menos, 15 anos, quando foi realizada a implantação da pastagem.

Os teores de fósforo observados no sistema plantio direto diferem de resultados amplamente observados na literatura. Falleiro et al. (2003) observaram incremento de fósforo no $\mathrm{PD}$ e, segundo estes autores, isto se deu em função da manutenção de resíduos de plantas na superfície do solo, neste sistema, o que favorece a ciclagem de fósforo, contribuindo para o incremento e disponibilidade dos estoques

Tabela 2. Atributos químicos, em função do uso e manejo do solo, no período de inverno (Dourados, MS, 2007).

\begin{tabular}{|c|c|c|c|c|c|c|c|c|c|c|}
\hline \multirow{2}{*}{ Manejo e uso do solo } & $\mathrm{pH}$ & MO & $\begin{array}{c}\mathrm{P} \\
\text { (Mehlich 1) }\end{array}$ & V & $\mathrm{m}$ & $\mathrm{Ca}$ & $\mathrm{K}$ & $\mathrm{Mg}$ & SB & CTC \\
\hline & $\left(\mathrm{CaCl}_{2}\right)$ & $\left(\mathrm{g} \mathrm{kg}^{-1}\right)$ & $\left(\mathrm{mg} \mathrm{dm}^{-3}\right)$ & \multicolumn{2}{|c|}{$-(\%)$} & \multicolumn{5}{|c|}{$-\mathrm{cmol}_{\mathrm{c}} \mathrm{dm}^{3}$} \\
\hline Vegetação nativa & $5,2 b^{*}$ & $31,9 \mathrm{ab}$ & $21,1 \mathrm{a}$ & $62,2 \mathrm{ab}$ & $0,4 \mathrm{~b}$ & $4,7 \mathrm{ab}$ & $0,37 \mathrm{~cd}$ & $3,1 \mathrm{a}$ & $8,1 \mathrm{bc}$ & $8,1 \mathrm{~b}$ \\
\hline Pastagem & $4,5 \mathrm{~d}$ & $29,5 \mathrm{~b}$ & $2,0 \mathrm{~b}$ & $35,8 \mathrm{c}$ & $19,0 \mathrm{a}$ & $2,3 \mathrm{c}$ & $0,26 \mathrm{~d}$ & $1,6 \mathrm{~b}$ & $4,0 \mathrm{c}$ & $4,9 \mathrm{c}$ \\
\hline Plantio direto & $5,8 \mathrm{a}$ & 33,9 a & $10,7 \mathrm{~b}$ & $75,6 \mathrm{a}$ & $0,0 \mathrm{~b}$ & $9,6 \mathrm{a}$ & $0,82 \mathrm{~b}$ & $3,1 \mathrm{a}$ & $13,5 \mathrm{a}$ & $13,5 \mathrm{a}$ \\
\hline Reflorestamento & $5,4 \mathrm{~b}$ & $30,9 \mathrm{ab}$ & $17,0 \mathrm{a}$ & $72,6 \mathrm{a}$ & $1,2 \mathrm{~b}$ & $6,5 a b$ & $1,11 \mathrm{a}$ & $2,6 \mathrm{ab}$ & $10,2 \mathrm{ab}$ & $10,2 \mathrm{ab}$ \\
\hline Sistema Convencional & $4,9 \mathrm{c}$ & $30,1 \mathrm{~b}$ & $16,7 \mathrm{a}$ & $54,8 \mathrm{~b}$ & $0,0 \mathrm{~b}$ & $4,1 \mathrm{bc}$ & $0,50 \mathrm{c}$ & $2,0 \mathrm{ab}$ & $6,7 \mathrm{bc}$ & $6,7 \mathrm{~b}$ \\
\hline $\mathrm{CV} \%$ & 4,4 & 18 & 27 & 16 & 100 & 50 & 21 & 37 & 43 & 41 \\
\hline
\end{tabular}

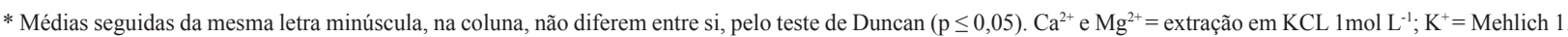


de fósforo, ao longo do tempo de plantio direto. De acordo com Sá (2004), o não revolvimento do solo, no sistema plantio direto, promove a formação de sítios de fósforo em espaçamentos e profundidades diferentes, devido à adubação fosfatada no sulco de semeadura.

Os menores valores de $\mathrm{pH}, \mathrm{CTC}$ e V(\%), observados no sistema pastagem $(\mathrm{P})$, também podem ser explicados pela ausência de calagem há 15 anos, bem como pelo avançado estado de degradação do sistema sob pastagem. Em geral, os solos do Cerrado apresentam baixa fertilidade natural e elevada acidez (Goedert 1989, Sousa \& Lobato 2004). Por isto, cerca de $80 \%$ das pastagens do Cerrado encontram-se sob algum grau de degradação (Barcellos 1996).

Quanto ao potássio, observa-se teor significativamente maior no reflorestamento $\left(1,11 \mathrm{cmol}_{\mathrm{c}} \mathrm{dm}^{-3}\right)$, seguido pelo sistema plantio direto $\left(0,82 \mathrm{cmol}_{\mathrm{c}}^{\mathrm{c}} \mathrm{dm}^{-3}\right)$. É possível que valores tão altos deste elemento estejam associados ao contínuo aporte de resíduos vegetais, que promoveram incremento na CTC, nestes dois sistemas (Tabela 2), favorecendo a retenção deste nutriente, além da ausência de revolvimento, que favorece o acúmulo de nutrientes na profundidade amostrada (Barreto et al. 2008). Ambos os sistemas apresentavam intensa cobertura sobre o solo.

Os diferentes sistemas de cultivo do solo não influenciaram, significativamente $(\mathrm{p} \leq 0,05)$, os atributos físicos do solo, quando comparados à vegetação nativa. Apesar de ter sido observada diferença entre os teores de matéria orgânica, em função dos diferentes usos ou manejos do solo, esta diferença não influenciou na densidade de partículas (Tabelas 2 e 3 ).

De forma semelhante aos resultados observados para densidade de partículas, não houve efeito significativo dos sistemas de manejo sobre a densidade do solo, que variou entre $1,10 \mathrm{~g} \mathrm{~cm}^{-3} \mathrm{e} 1,14 \mathrm{~g} \mathrm{~cm}^{-3}$. Segundo Brady \& Buckman (1983), um fator que contribui para a redução da densidade média das partículas (DP) e densidade do solo (DS), além de afetar a agregação, é o teor de matéria orgânica do solo. Entretanto, neste estudo, não houve correlação significativa entre estas variáveis (Tabelas 2 e 4).

De forma semelhante, os sistemas não promoveram diferenças nos valores de volume total de poros (VTP) e diâmetro médio ponderado dos agregados (DMP). É importante considerar que o Latossolo Vermelho distroférrico típico, por sua mineralogia oxídica, deve apresentar estrutura mais resistente ao rompimento pela agitação mecânica (Vitorino et al. 2003).

Os resultados observados para DMP discordam de pesquisas em que se observou efeito dos sistemas de manejo sobre os valores da densidade do solo, havendo incremento nos valores desta variável, com a adoção do plantio direto (Tormena et al. 2002, Araújo et al. 2004). É possível que a recente implantação dos sistemas de cultivo, associada aos

Tabela 3. Atributos físicos, em função do uso e manejo do solo (Dourados, MS, 2007).

\begin{tabular}{lcccccccc}
\hline \multirow{2}{*}{ Manejo e uso do solo } & DP & DS & VTP & DMP & Areia & Silte & Argila \\
\cline { 2 - 6 } Classe de solo** & $\left(\mathrm{g} \mathrm{cm}^{-3}\right)$ & $\left(\mathrm{g} \mathrm{cm}^{-3}\right)$ & $(\%)$ & $(\mathrm{mm})$ & & & $(\%)$ & \\
Vegetação nativa & $2,77 \mathrm{a}^{*}$ & $1,14 \mathrm{a}$ & $58,77 \mathrm{a}$ & $0,52 \mathrm{a}$ & $12,94 \mathrm{a}$ & $14,2 \mathrm{a}$ & $72,8 \mathrm{a}$ & LVdf \\
Pastagem & $2,65 \mathrm{a}$ & $1,14 \mathrm{a}$ & $56,95 \mathrm{a}$ & $0,48 \mathrm{a}$ & $8,62 \mathrm{c}$ & $24,3 \mathrm{a}$ & $67,0 \mathrm{a}$ & LVdf \\
Plantio direto & $2,72 \mathrm{a}$ & $1,12 \mathrm{a}$ & $58,85 \mathrm{a}$ & $0,69 \mathrm{a}$ & $9,42 \mathrm{c}$ & $18,9 \mathrm{a}$ & $71,6 \mathrm{a}$ & LVdf \\
Reflorestamento & $2,77 \mathrm{a}$ & $1,11 \mathrm{a}$ & $59,82 \mathrm{a}$ & $0,65 \mathrm{a}$ & $10,93 \mathrm{~b}$ & $17,3 \mathrm{a}$ & $71,8 \mathrm{a}$ & LVdf \\
Sistema Convencional & $2,63 \mathrm{a}$ & $1,10 \mathrm{a}$ & $57,99 \mathrm{a}$ & $0,70 \mathrm{a}$ & $13,59 \mathrm{a}$ & $25,1 \mathrm{a}$ & $61,3 \mathrm{a}$ & LVdf \\
\hline CV \% & 2,0 & 2,8 & 2,3 & 2,4 & 5,7 & 18 & 5,0 & \\
\hline
\end{tabular}

* Médias seguidas da mesma letra minúscula, na coluna, não diferem entre si, pelo teste de Duncan $(\mathrm{p} \leq 0,05)$ ** LVdf $=$ Latossolo Vermelho distroférrico; DP = densidade de partículas; DS = densidade do solo; VTP = volume total de poros; DMP = diâmetro médio ponderado.

Tabela 4. Coeficientes de correlação de Pearson entre atributos químicos, físicos e microbiológicos do solo submetido a diferentes condições de uso e manejo (Dourados, MS, 2007).

\begin{tabular}{|c|c|c|c|c|c|c|c|c|c|c|c|c|c|c|}
\hline & $\mathrm{DP}$ & DS & $\mathrm{PT}$ & DMP & $\mathrm{PH}$ & $\mathrm{AL}$ & $\mathrm{K}$ & $\mathrm{P}$ & $\mathrm{Ca}$ & $\mathrm{Mg}$ & $\mathrm{SB}$ & CTC & $\mathrm{V}$ & $\mathrm{M} \%$ \\
\hline $\mathrm{MO}$ & ns & ns & $\mathrm{ns}$ & ns & $0,46^{*}$ & $-0,50 *$ & ns & 0,41 & ns & $0,33 * *$ & ns & $0,43 * *$ & $0,40 * *$ & $-0,51 * *$ \\
\hline C-BMS & $0,34 * *$ & $0,41 * *$ & ns & $0,42 * *$ & ns & ns & $-0,37 * *$ & ns & ns & $\mathrm{ns}$ & ns & ns & ns & ns \\
\hline $\mathrm{C}-\mathrm{CO}_{2}$ & $0,53 * *$ & $0,37 * *$ & ns & $-0,36 * *$ & ns & ns & ns & $0,47^{*}$ & ns & ns & ns & ns & ns & ns \\
\hline$q \mathrm{CO}_{2}$ & $0,52 *$ & ns & ns & ns & ns & $-0,38 * *$ & ns & $0,67^{*}$ & $\mathrm{~ns}$ & ns & ns & ns & ns & $-0,36 * *$ \\
\hline$q \mathrm{MIC}$ & ns & ns & ns & ns & ns & ns & ns & ns & ns & ns & ns & ns & ns & ns \\
\hline
\end{tabular}


dez anos de semeadura direta, antes da implantação dos sistemas de cultivo, não tenha sido suficiente para prover diferenças significativas nestes atributos físicos do solo.

A adoção do sistema convencional de preparo do solo, em função do revolvimento, normalmente favorece uma redução nos valores do DMP. De forma semelhante, Bertol et al. (2001), ao estudarem esta variável, em diferentes profundidades, não observaram efeito do manejo sobre a mesma, porém, observaram tendência de redução no diâmetro dos agregados, à medida que se aumentava a profundidade. Segundo estes autores, a redução do diâmetro se deu em função da redução no teor de matéria orgânica, isto porque ela desempenha uma importante função na agregação e estabilidade de agregados.

Neste trabalho, não foi observado efeito do manejo e uso do solo no VTP, no primeiro ano de revolvimento de um sistema de semeadura direta. Entretanto, segundo Schaefer et al. (2001), o efeito do preparo sobre os valores de porosidade do solo pode ser pouco evidente, sendo mais comuns os efeitos na forma e distribuição dos poros, ao longo do perfil do solo.

\section{Correlação de Pearson}

Por meio do estudo da correlação de Pearson, foi possível observar correlação negativa e significativa entre C-BMS e teor de $\mathrm{K}$ no solo e positiva e significativa entre respiração basal $\left(\mathrm{C}-\mathrm{CO}_{2}\right)$ e teor de $\mathrm{P}$. $\mathrm{O} q \mathrm{CO}_{2}$ correlacionou-se significativamente e de forma positiva com o teor de $\mathrm{P}$ e negativa com o teor de Al (Tabela 4).

A biomassa microbiana foi, significativamente, influenciada pelos teores de K no solo, de forma que, quanto maior o teor de K, menor o C-BMS. É possível que os altos teores deste elemento no solo $\left(>0,204 \mathrm{cmol}_{c} \mathrm{dm}^{-3}\right)$ tenham exercido efeito supressor ao desenvolvimento da biomassa microbiana do solo (Tabela 2) (Sousa \& Lobato 2004).

A respeito da correlação positiva do teor de $\mathrm{P}$ no solo com os teores de $\mathrm{C}-\mathrm{CO}_{2}$ emitidos, Baretta et al. (2008) observaram que mudanças químicas pouco perceptíveis nos valores de P no solo podem afetar atributos microbianos. Na literatura, há relatos de que os teores deste nutriente no solo podem limitar a biomassa microbiana, em algumas situações, e, em outras, a adição de $\mathrm{P}$ pode exercer efeito benéfico (Wardle \& Hungria 1994, Maluche-Baretta et al.
2006). Baretta et al. (2008) observaram que o teor de $\mathrm{P}$ no solo está diretamente relacionado com os atributos microbianos, tendo uma relação positiva com os atributos $\mathrm{CBM}$ e $\mathrm{C}-\mathrm{CO}_{2}$.

A correlação positiva e significativa entre teor de $\mathrm{P}$ no solo e $q \mathrm{CO}_{2}$ indica que, quanto maior o teor de $\mathrm{P}$ do solo, maior serão os valores do $q \mathrm{CO}_{2} \mathrm{e}$, portanto, altos teores deste nutriente, nas condições deste estudo, podem favorecer uma condição de estresse ou de distúrbio ao sistema (Tótola \& Chaer 2002). Segundo Gama-Rodrigues (1999), menores valores desta variável são indicativos de uma biomassa microbiana mais eficiente, ou seja, com menor perda de carbono como $\mathrm{CO}_{2}$, pela respiração, e com uma fração significativa de $\mathrm{C}$ sendo incorporada ao tecido microbiano.

Quanto maior a perda de carbono do solo, na forma de $\mathrm{CO}_{2}$, maior será a DS e menor o DMP, o que ressalta a importância do uso de práticas conservacionistas de manejo como a sucessão, rotação de culturas e o cultivo mínimo, ou, até mesmo, o plantio direto (Balota et al. 1998, Alovisi et al. 2007).

\section{CONCLUSÕES}

1. A avaliação do sistema de manejo, em seu primeiro ano de implantação, não foi suficiente para mensurar os impactos sobre atributos físicos e microbiológicos do solo.

2. A substituição da vegetação nativa por sistemas de cultivo pode causar importantes alterações nos atributos químicos do solo, já no primeiro ano de implantação.

3. Os atributos microbiológicos foram eficientes indicadores de alterações nos atributos físicos, em função do manejo e uso do solo.

\section{REFERÊNCIAS}

ALEXANDER, M. Introduction to soil microbiology. 2. ed. New York: John Willey \& Sons, 1977.

ALOVISI, A. M. T. et al. Alterações de atributos físicos e químicos de solo sob sistemas de manejo em Dourados MS. In: CONGRESSO BRASILEIRO DE CIÊNCIA DO SOLO, 31., 2007, Gramado. Anais... Gramado: SBCS, 2007. p. 1-4.

ALVARENGA, M. I. N.; SIQUEIRA, J. O.; DAVIDE, A. C. Teor de carbono, biomassa microbiana, agregação e micorriza em solos de Cerrado com diferentes usos. 
Ciência e Agrotecnologia, Lavras, v. 23, n. 3, p. 617-625, 1999.

ALVAREZ, R.; SANTANATOGLIA, O. J.; GARCIA, R. Effect of temperature on soil microbial biomass and its metabolic quotient in situ under different tillage systems. Biology and Fertility of Soils, Berlin, v. 19, n. 2-3, p. 227230, 1995.

ARAÚJo, M. A.; TORMENA, C. A.; SILVA, A. P. Propriedades físicas de um Latossolo Vermelho distrófico cultivado sob mata nativa. Revista Brasileira de Ciência do Solo, Viçosa, v. 28, n. 2, p. 337-345, 2004.

ARAÚJO, R.; GOEDERT, W. J.; LACERDA, M. P. C. Qualidade de um solo sob diferentes usos e sob Cerrado nativo. Revista Brasileira de Ciência do Solo, Viçosa, v. 31, n. 5, p. 1099-1108, 2007.

AYOADE, J. O. Introdução à climatologia para os trópicos. São Paulo: Difel, 1986.

BALOTA, E. L. et al. Biomassa microbiana e sua atividade em solos sob diferentes sistemas de preparo e sucessão de culturas. Revista Brasileira de Ciência do Solo, Viçosa, v. 22, n. 4, p. 641-649, 1998.

BALOTA, E. L. et al. Microbial biomass in soils under different tillage and crop rotation systems. Biology and Fertility of Soils, Berlin, v. 38, n. 1, p. 15-20, 2003.

BARCELLOS, A. de O. Sistemas extensivos e semiextensivos de produção: pecuária bovina de corte nos Cerrados. In: SIMPÓSIO SOBRE O CERRADO, 8.; INTERNACIONAL SYMPOSIUM ON TROPICAL SAVANNAS, 1., 1996, Brasília, DF. Anais... Planaltina, DF: Embrapa-CPAC, 1996. p. 130-136.

BARETTA, D.; MALUCHE-BARETTA, C. R. D.; CARDOSO, E. J. B. N. Análise multivariada de atributos microbiológicos e químicos do solo em florestas com Araucaria angustifólia. Revista Brasileira de Ciência do Solo, Viçosa, v. 32, n. esp., p. 2683-2691, 2008.

BARRETO, P. A. B. et al. Atividade microbiana, carbono e nitrogênio da biomassa microbiana em plantações de eucalipto, em sequência de idades. Revista Brasileira de Ciência do Solo, Viçosa, v. 32, n. 2, p. 611-619, 2008.

BAYER, C.; MIELNICZUK, J. Dinâmica e função da matéria orgânica. In: SANTOS, G. A.; CAMARGO, F. A. O. Fundamentos da matéria orgânica do solo: ecossistemas tropicais e subtropicais. Porto Alegre: Gênesis, 1999. p. 9-26.

BENINTENDE, S. M. et al. Soil microbiological indicators of soil quality in four rice rotation systems. Ecological Indicators, Amsterdam, v. 8, n. 5, p. 704-708, 2008.

BERTOL, I. et al. Propriedades físicas de um Cambissolo húmico afetadas pelo tipo de manejo do solo. Scientia Agricola, Piracicaba, v. 58, n. 3, p. 555-560, 2001.
BLAKE, G. R.; HARTGE, K. H. Bulk density. In: KLUTE, A. (Ed.). Methods of soil analysis: physical and mineralogical methods. Madison: ASA, 1986. p. 363-375.

BRADY, N. C.; BUCKMAN, H. O. Natureza e propriedades dos solos. 6. ed. Rio de Janeiro: Freitas Bastos, 1983.

CALEGARI, A. Coberturas verdes em sistemas intensivos de produção. In: WORKSHOP NITROGÊNIO NA SUSTENTABILIDADE DE SISTEMAS INTENSIVOS DE PRODUÇÃO AGROPECUÁRIA, 1., 2000, Dourados. Anais... Dourados: Embrapa Agropecuária Oeste, 2000. p. 141-153.

CARNEIRO, M. A. C. et al. Atributos físicos, químicos e biológicos de solo de Cerrado sob diferentes sistemas de uso e manejo. Revista Brasileira de Ciência do Solo, Viçosa, v. 33, n. 1, p. 147-157, 2009.

CLAESSEN, M. E. C. (Org.). Manual de métodos de análise de solo. 2. ed. rev. atual. Rio de Janeiro: EmbrapaCNPS, 1997. (Documentos, 1).

CORREIA, M. E. F.; ANDRADE, A. G. de. Formação de serapilheira e ciclagem de nutrientes. In: SANTOS, G. A.; CAMARGO, F. A. O. (Eds.). Fundamentos da matéria orgânica do solo: ecossistemas tropicais e subtropicais. Porto Alegre: Gênesis, 1999. p. 227-243.

COSTA, E. A.; GOEDERT, W. J.; SOUZA, D. M. G. de. Qualidade de solo submetido a sistemas de cultivo com preparo convencional e plantio direto. Pesquisa Agropecuária Brasileira, Brasília, DF, v. 41, n. 7, p. 11851191, 2006.

D`ANDRÉA, A. F. et al. Atributos biológicos indicadores da qualidade do solo em sistema de manejo na região do Cerrado no sul do Estado de Goiás. Revista Brasileira de Ciências do Solo, Viçosa, v. 26, n. 4, p. 913-923, 2002.

DORAN, J. W.; PARKIN, T. B. Defining and assessing soil quality. In: DORAN, J. W. et al. (Eds.). Defining soil quality for a sustainable environment. Madison: Soil Science Society of America, 1994. p. 3-22.

ESPÍNDOLA, J.A. A. et al. Flutuação sazonal da biomassa microbiana e teores de nitrato e amônio de solo coberto com Paspalum notatum em um agroecossistema. Floresta e Ambiente, Rio de Janeiro, v. 8, n. 1, p. 104-113, 2001.

FALLEIRO, R. M. et al. Influência dos sistemas de preparo nas propriedades químicas e físicas do solo. Revista Brasileira de Ciência do Solo, Viçosa, v. 27, n. 6, p. 1097-1104, 2003.

GAMA-RODRIGUES, E. F. da. Biomassa microbiana e ciclagem de nutrientes. In: SANTOS, G. de A.; CAMARGO, F. A. de O. Fundamentos da matéria 
orgânica do solo: ecossistemas tropicais e subtropicais. Porto Alegre: Gênesis, 1999. p. 227-243.

GOEDERT, W. J. Região dos Cerrados: potencial agrícola e política para seu desenvolvimento. Pesquisa Agropecuária Brasileira, Brasília, DF, v. 24, n. 1, p. 1-17, 1989.

LOURENTE, E. R. P. Manejo do solo e suas influências em atributos químicos e microbiológicos do solo. 2007. $66 \mathrm{f}$. Tese (Doutorado em Agronomia)-Universidade Federal da Grande Dourados, Dourados, 2007.

MALUCHE-BARETTA, C. R. D.; AMARANTE, C. V. T.; KLAUBERG FILHO, O. Análise multivariada de atributos do solo em sistemas convencional e orgânico de produção de maçãs. Pesquisa Agropecuária Brasileira, Brasília, DF, v. 41, n. 10, p. 1531-1539, 2006.

MARCHIORI JÚNIOR, M.; MELO, W. J. Alterações na matéria orgânica e na biomassa microbiana em solo de mata natural submetido a diferentes manejos. Pesquisa Agropecuária Brasileira, Brasília, DF, v. 35, n. 6, p. 1177 $1182,2000$.

MATSUOKA, M.; MENDES, I. C.; LOUREIRO, M. F. Biomassa microbiana e atividade enzimática em solos sob vegetação nativa e sistemas agrícolas anuais e perenes na região de Primavera do Leste (MT). Revista Brasileira de Ciências do Solo, Viçosa, v. 27, n. 3, p. 425-433, 2003.

MERCANTE, F. M. Os micro-organismos do solo e a dinâmica da matéria orgânica em sistema de produção de grãos e pastagem. Dourados: Embrapa Agropecuária Oeste, 2001. (Sistema plantio direto, 5).

PIAO, H. C.; HONG, Y. T.; YUAN, Z. Y. Seasonal changes of microbial biomass carbon related to climatic factors in soils from Karst areas of southwest China. Biology and Fertility of Soils, Berlin, v. 30, n. 4, p. 294-297, 2000.

RIBEIRO JÚNIOR, J. I. Análises estatísticas no SAEG. Viçosa: Ed. da UFV, 2001.

SÁ, J. C. de M. Adubação fosfatada no sistema plantio direto. In: YAMADA, T.; ABDALLA, S. R. S. e (Eds.). SIMPÓSIO SOBRE FÓSFORO NA AGRICULTURA BRASILEIRA, 1., 2003, São Pedro. Anais... Piracicaba: Potafos, 2004. p. 201-222.

SAMPAIO, D. B.; ARAÚJO, A. S. F. de; SANTOS, V. B. dos. Avaliação de indicadores biológicos de qualidade do solo sob sistemas de cultivo convencional e orgânico de frutas. Ciência e Agrotecnologia, Lavras, v. 32, n. 2, p. 353-359, 2008.
SCHAEFER, C. E. G. R. et al. Características da porosidade de um Argissolo Vermelho-Amarelo submetido a diferentes sistemas de preparo de solo. Revista Brasileira de Ciência do Solo, Viçosa, v. 25, n. 6, p. 765-769, 2001.

SOUSA, D. M. G.; LOBATO, E. Correção da acidez do solo. In: SOUSA, D. M. G.; LOBATO, E. (Eds.). Cerrado: correção do solo e adubação. Brasília, DF: Embrapa, 2004. p. 81-95.

TORMENA, C. A. et al. Densidade, porosidade e resistência à penetração em Latossolo cultivado sob diferentes sistemas de preparo do solo. Scientia Agricola, Piracicaba, v. 59, n. 4, p. 795-801, 2002.

TÓTOLA, M. R.; CHAER, G. M. Micro-organismos e processos microbiológicos como indicadores da qualidade dos solos. Tópicos em Ciências do Solo, Viçosa, v. 2, n. 2, p. 195-276, 2002.

VANCE, E. D.; BROOKES, P. C.; JENKINSON, D. S. An extraction method for measuring soil microbial biomass-C. Soil Biology \& Biochemistry, Amsterdam, v. 19, n. 6, p. 703-707, 1987.

VIEIRA, M. J.; MUZILLI, O. Características físicas de um Latossolo Vermelho-Escuro sob diferentes sistemas de manejo. Pesquisa Agropecuária Brasileira, Brasília, DF, v. 19, n. 7, p. $873-882,1984$.

VITORINO, A. C. T. et al. Mineralogia, química e estabilidade de agregados do tamanho de silte de solos da Região Sudeste do Brasil. Pesquisa Agropecuária Brasileira, Brasília, DF, v. 38, n. 1, p. 133-141, jan. 2003.

WARDLE, D. A.; HUNGRIA, M. A. A biomassa microbiana do solo e sua importância nos ecossistemas terrestres. In: ARAÚJO, R. S.; HUNGRIA, M. A. (Eds.). Micro-organismos de importância agrícola. Brasília, DF: Embrapa-SPI, 1994. p. 193-216.

YEOMANS, J. C.; BREMNER, J. M. A rapid and precise method for routine determination of organic carbon in soil. Communications in Soil Science and Plant Analyses, Philadelphia, v. 19, n. 13, p. 1467-1476, 1989. 